\title{
Transvenous Embolization of Cavernous and Paracavernous Dural Arteriovenous Fistula through the Facial Vein: Report of 12 Cases
}

\author{
Myeong Jin Kim, MD', Yong Sam Shin, MD, PhD', Yon Kwon Ihn, MD², Byung Moon Kim, MD, \\ Pyeong Ho Yoon, MD, Se-yang Oh, MD5, Bum-soo Kim, MD, PhD
}

Purpose: The aim of this study was to evaluate the feasibility and safety of the transfacial venous embolization of cavernous or paracavernous dural arteriovenous fistula (DAVF) in which approach via inferior petrosal sinus (IPS) was not feasible.

Materials and Methods: We identified the cases of transfacial venous embolization of cavernous sinus (CS) or adjacent dural sinuses from the neurointerventional database of three hospitals. The causes and clinical and angiographic outcomes of transfacial venous embolization were retrospectively evaluated.

Results: Twelve patients with CS ( $n=11)$ or lesser wing of sphenoid sinus (LWSS, $n=1)$ DAVF were attempted to treat by transvenous embolization via ipsilateral $(n=10)$ or contralateral $(n=2)$ facial vein. Trans-IPS access to the target lesion was impossible due to chronic occlusion $(n=11)$ or acute angulation adjacent the target lesion $(n=1)$. In all twelve cases, it was possible to navigate through facial vein, angular vein, superior ophthalmic vein, and then CS. It was also possible to further navigation to contralateral CS through intercavernous sinus in two cases, and laterally into LWSS in one case. Post-treatment control angiography revealed complete occlusion of the DAVF in eleven cases and partial occlusion in one patient, resulting in complete resolution of presenting symptom in eight and gradually clinical improvement in four patients. There was no treatment-related complication during or after the procedure.

Conclusion: In the cavernous or paracavernous DAVF in which trans-IPS approach is not feasible, the facial vein seems to be safe and effective alternative route for transvenous embolization.

Key Words : Arteriovenous fistula; Cavernous sinus; Endovascular; Facial vein

\footnotetext{
'Department of Neurosurgery and ${ }^{5}$ Radiology, Seoul St. Mary's Hospital, The Catholic University of Korea, Seoul, Korea

${ }^{2}$ Department of Radiology, St. Vincent's Hospital, The Catholic University of Korea, Suwon, Korea

${ }^{3}$ Department of Radiology, Severance Hospital, Yonsei University College of Medicine, Seoul, Korea

${ }^{4}$ Department of Radiology, NHIC Ilsan Hospital of Korea, Ilsan, Korea

Received November 16, 2012; accepted after revision December 14, 2012.

Correspondence to: Bum-soo Kim, MD, PhD, Department of Radiology, Seoul St. Mary's Hospital, The Catholic University of Korea, 505, Banpo-dong, Seocho-gu, Seoul 137-701, Korea.

Tel. 82.2.2258.6239 Fax.82.2.599.6771 E-mail: bumrad@catholic.ac.kr

This is an Open Access article distributed under the terms of the Creative Commons Attribution Non-Commercial License (http://creativecommons.org/licenses/by-nc/3.0) which permits unrestricted non-commercial use, distribution, and reproduction in any medium, provided the original work is properly cited.
} 
Transvenous endovascular embolization is widely accepted to be an effective and safe treatment option for cavernous dural arteriovenous fistula (DAVF). Specific transvenous route for embolization may depends on the compartment of the cavernous sinus (CS) involved in the fistula and its venous drainage [1, 2]. The venous route goes usually through the internal jugular vein (IJV) and the inferior petrosal sinus (IPS) up to the pathologic shunts at or adjacent to the CS, but this approach is occasionally not feasible because of chronic occlusion of the IPS [3] or geographically tortuous route to the target lesion through the IPS. In this impregnable case, some authors have proposed navigation through the obstructed IPS [4] or a superior ophthalmic vein (SOV) approach, mostly performed through surgical exposure [5-7]. These challengeable techniques might be not always practicable, and moreover some considerable complications of transvenous approaches through the occluded IPS or by the cut-down on the SOV have been reported $[2,8,9]$. In selected cases, facial vein (FV) could be an effective alternative route for successful obliteration of cavernous DAVF with impregnable trans-IPS approach [3]. The aim of this study was to evaluate the feasibility and safety of the trans-facial venous embolization of cavernous or paracavernous DAVF in which trans-IPS approach was not feasible.

\section{MATERIALS AND METHODS}

Between 1996 and 2011, all patients who underwent endovascular treatment via facial venous embolization for DAVF were identified from the neurointerventional database of three hospitals. Clinical outcomes and radiologic findings of all patients with DAVF treated with transvenous embolization via FV were retrospectively reviewed. All patients underwent the endovascular treatment under general anesthesia. The dosage of heparin was intravenously 2,000 or 3,000 IU bolus at the beginning of the procedure and if the procedure prolonged more than one hour, additional heparin 1,000 IU or more dose was administrated intravenously after checking activated clotting time. Venous approach was performed through the IJV to the FV in all procedure. The 5 or 6 Fr Envoy guiding catheters (Codman \& Shurtleff, Rayham, MA, USA) were placed at to the level of mandibular angle of the FV in all cases.

\section{RESULTS}

Twelve patients with CS $(\mathrm{n}=11)$ or lesser wing of sphenoid sinus (LWSS, $n=1$ ) DAVF were attempted to treat by transvenous embolization via ipsilateral $(\mathrm{n}=$ $10)$ or contralateral $(n=2) \mathrm{FV}$. All procedures were successful. The patient group consisted of six male and six female patients with an age range from 20 to 83 years (mean age, 55 years). Clinical findings included

Table 1. Summarized Symptom, Angiographic Result and Clinical Outcome of Twelve Patients with Cavernous Type DAVF Treated Endovascular Embolization

\begin{tabular}{rllll}
\hline No. & Sex/Age & Symptom & Angiographic Result & Clinical Outcome \\
\hline 1 & $\mathrm{~F} / 59$ & Exophthalmos & Complete remission & Improved \\
\hline 2 & $\mathrm{~F} / 75$ & Exophthalmos & Complete remission & Improved \\
\hline 3 & $\mathrm{~F} / 42$ & Exophthalmos & Complete remission & Improved \\
\hline 4 & $\mathrm{M} / 54$ & Exophthalmos, seizure & Complete remission & Improved \\
\hline 5 & $\mathrm{M} / 20$ & Exophthalmos, chemosis & Complete remission & Improved \\
\hline 7 & $\mathrm{~F} / 83$ & Exophthalmos, diplopia & Complete remission & Diplopia remained \\
\hline 9 & $\mathrm{M} / 61$ & Exophthalmos, diplopia & Incomplete remission & Diplopia remained \\
\hline 10 & $\mathrm{M} / 46$ & Exophthalmos & Complete remission & Improved \\
\hline 11 & $\mathrm{~F} / 53$ & Exophthalmos, diplopia & Complete remission & Improved \\
\hline 12 & $\mathrm{M} / 55$ & Diplopia & Complete remission & Diplopia remained \\
\hline
\end{tabular}

Abbreviations: DAVF, dural arteriovenous fistula; $F$, female; $M$, male 


\section{Embolization of Cavernous Dural Arteriovenous Fistula}

exophthalmos and chemosis $(\mathrm{n}=10)$, limitation of lateral gaze in ipsilateral eye $(\mathrm{n}=3)$, headache $(\mathrm{n}=1)$, tinnitus $(\mathrm{n}=1)$ and intracranial hemorrhage $(\mathrm{n}=1)$. The characteristics of each DAVF are summarized in Table 1 and 2. Trans-IPS access to the target lesion was impossible due to chronic occlusion ( $\mathrm{n}=11)$ of the IPS or acute angulated route adjacent the target lesion ( $\mathrm{n}=$ 1). In all twelve cases, it was possible to catheterize the FV with guiding catheter, and to navigate the microcatheter through the angular vein and SOV, and then to reach the CS. Eleven of all cases had thrombosed IPS, so it was not accessible to approach the shunt through the route of IPS. In one case, the IPS was patent, but the attempt to reach the shunt via this IPS was failed due to acute angle between CS and
LWSS. The procedure for this case is more described in Case 2.

Postembolization control angiography revealed complete occlusion of the shunt in eleven patients and partial occlusion in one patient. Procedure-related complications were not observed in any patients. The exophthalmos and chemosis in all ten patients improved after the procedure, but sixth nerve palsy in three patients was not improved immediately but improved slightly at two months during clinical follow-up.

\section{Representative Cases}

\section{Case 1 (patient 4 in table)}

A 55-year-old man was admitted with dysarthria, left
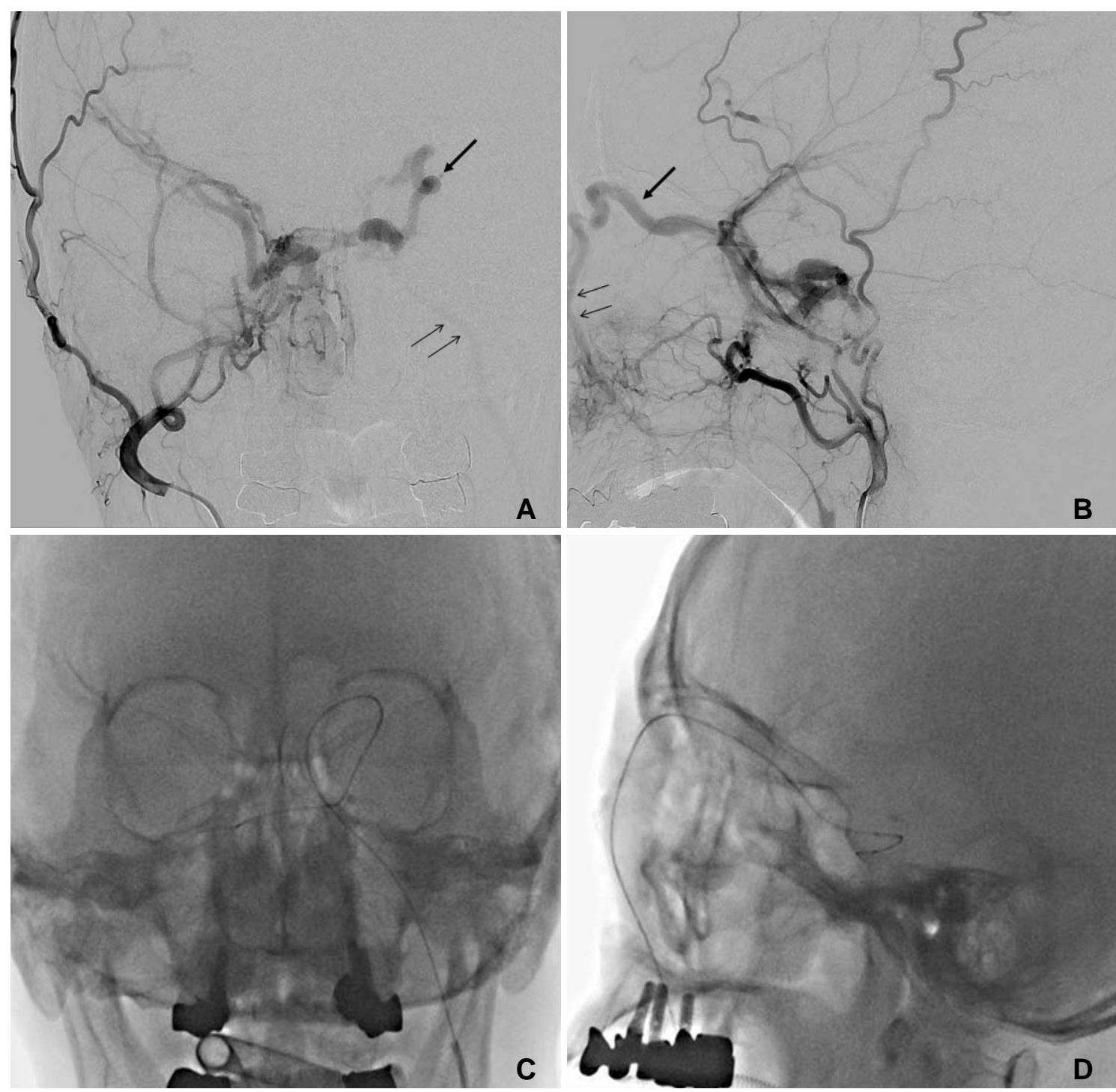

Fig. 1. Antero-posterior $(\mathbf{A})$ and lateral $(\mathbf{B})$ view of right ECA angiography show DAVF involving CS with arterial feeder from ECA and venous drainage to left SOV (large arrow), right sphenoparietal sinus and superficial middle cerebral vein through intercavernous sinus. (C, D) Through left facial vein (small arrows in figure $\mathbf{A}$ and $\mathbf{B}$ ), the microwire was placed in right CS beyond the intercavernous sinus. 

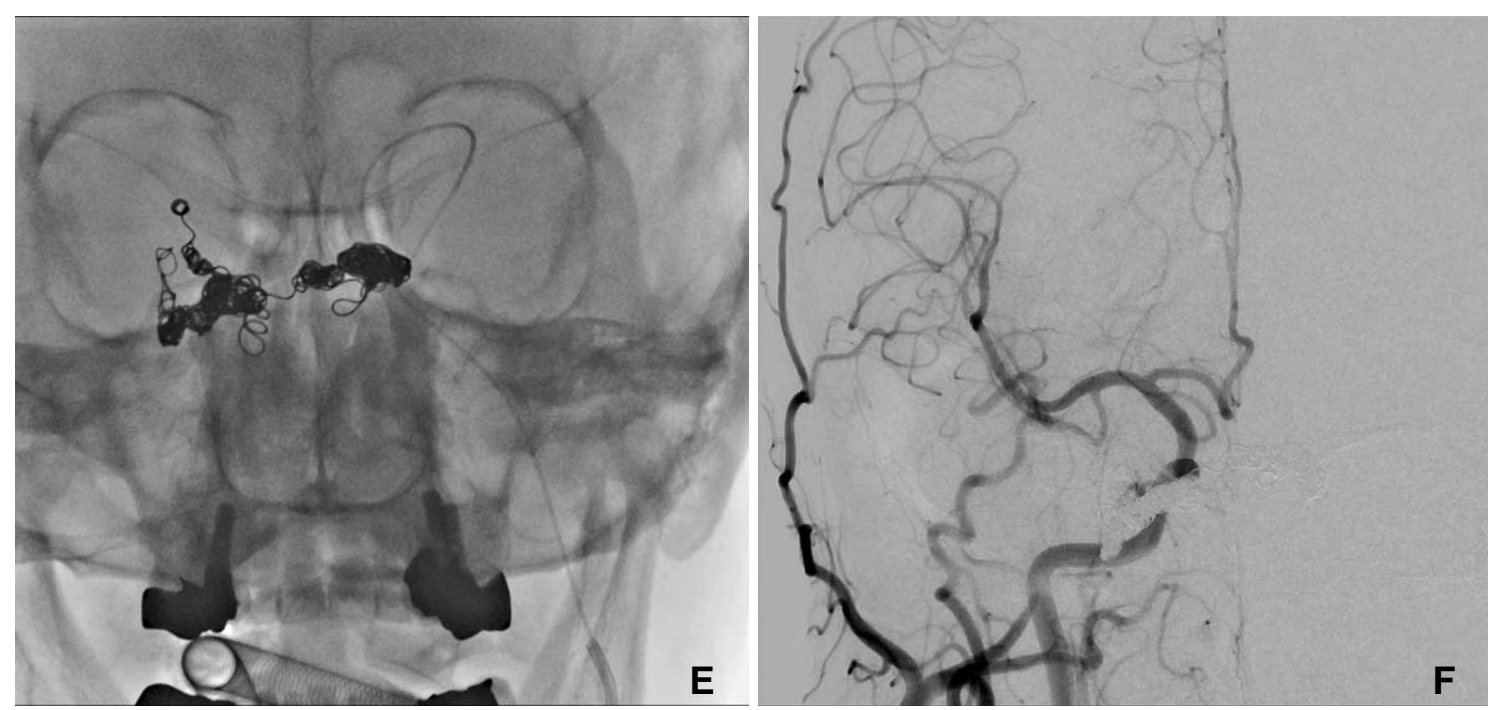

Fig. 1. (E) Coil embolization was performed for the shunt lesion using detachable and pushable fibered coils. (F) After the procedure, antero-posterior view of angiography selected right common carotid artery shows complete occlusion of the shunt.

Table 2. Angiographic Characteristics of Twelve Patients with Cavernous Type DAVF

\begin{tabular}{cllll}
\hline No. & Feeder & Shunt & Drained vein & IPS \\
\hline 1 & Rt ICA / Rt ECA & Rt CS & InterCS-Lt SOV & Occluded \\
2 & Rt ICA / Rt ECA & Rt CS & Rt SOV & Occluded \\
3 & Rt ICA / Rt ECA & Rt CS & Rt SOV & Occluded \\
4 & Rt ICA / Rt ECA & Rt CS & Rt SMCV/InterCS-Lt CS-Lt SOV & Occluded \\
\hline 5 & Lt ICA / Lt ECA & LWSS & Lt SOV/Lt IPS & Opened \\
\hline 6 & Lt ICA / Lt ECA & Lt CS & Lt SOV & Occluded \\
7 & Lt ICA / Lt ECA & Lt CS & Lt SOV/Lt SPS/Lt SMCV & Occluded \\
8 & Lt ICA / Lt ECA & Lt CS & Lt SOV & Occluded \\
\hline 9 & Both ICA / Both ECA & Both CS & Both SOV & Occluded \\
\hline 10 & Both ICA / Lt ECA & Lt CS & Lt SOV & Occluded \\
11 & Lt ICA / Lt ECA & Lt CS & Lt SOV & Occluded \\
12 & Rt ICA / Rt ECA & Rt CS & Rt SOV & Occluded \\
\hline
\end{tabular}

Abbreviations: ICA, internal carotid artery; ECA, external carotid artery; CS, avernous sinus; DAVF, dural arteriovenous fistula; SOV, superior ophthalmic vein; Lt, left; LWSS, lesser wing of sphenoid sinus; Rt, right; SMCV, superficial middle cerebral vein; SPS, superior petrosal sinus

exophthalmos, chemosis and left-side motor weakness. Brain computed tomographic angiography (CTA) revealed acute intracranial hemorrhage in right capsular region and tangled vessels of right temporal area with early venous drainage. Digital subtraction angiography (DSA) showed DAVF involving CS with arterial feeders from right external carotid artery (ECA) and internal carotid artery (ICA), venous drainage to left SOV, right sphenoparietal sinus and superficial middle cerebral vein, thought as dangerous retrograde cortical drainage, through intercavernous sinus (Fig. 1). We decided to occlude the fistula by coil embolization via venous route. Under general anesthesia, initially a guiding catheter was placed in the left IJV to FV and a microcatheter - exchanged Prowler select plus to Excelsior SL-10 straight because of tortuous angular vein - could be introduced in left CS through SOV by a microwire (Transend 0.014, Boston Scientific, Natick, MA, USA). However, the microwire and microcatheter could not be passed through intercavernous sinus, so 


\section{Embolization of Cavernous Dural Arteriovenous Fistula}

the microwire was changed to Agility 14 Soft microwire (Codman \& Shurtleff, Rayham, MA, USA). The microcatheter was then exchanged with Prowler select plus (Codman, USA), and it was advanced to the transition site between the right CS and sphenoparietal sinus (Fig. 1). The fistula site in the CS was completely occluded by embolization using detachable and pushable fibered coils through from right to left CS involving intercavernous portion. The patient discharged with no neurological deficit.

\section{Case 2 (patient 5 in table)}

A 20-year-old man was admitted with left-sided exophthalmos and chemosis. Enhanced orbit MRI revealed a lobulated mass-like lesion at the left sphenoid bone with extension to the orbit and middle cranial fossa, and it showed strong enhancement. DSA showed an arterio-venous fistula involving the left frontal region, with shunt flow from the middle meningeal artery, draining into the dilated venous, and then into LWSS and SOV through CS (Fig. 2). We decided to occlude the fistula portion involving middle
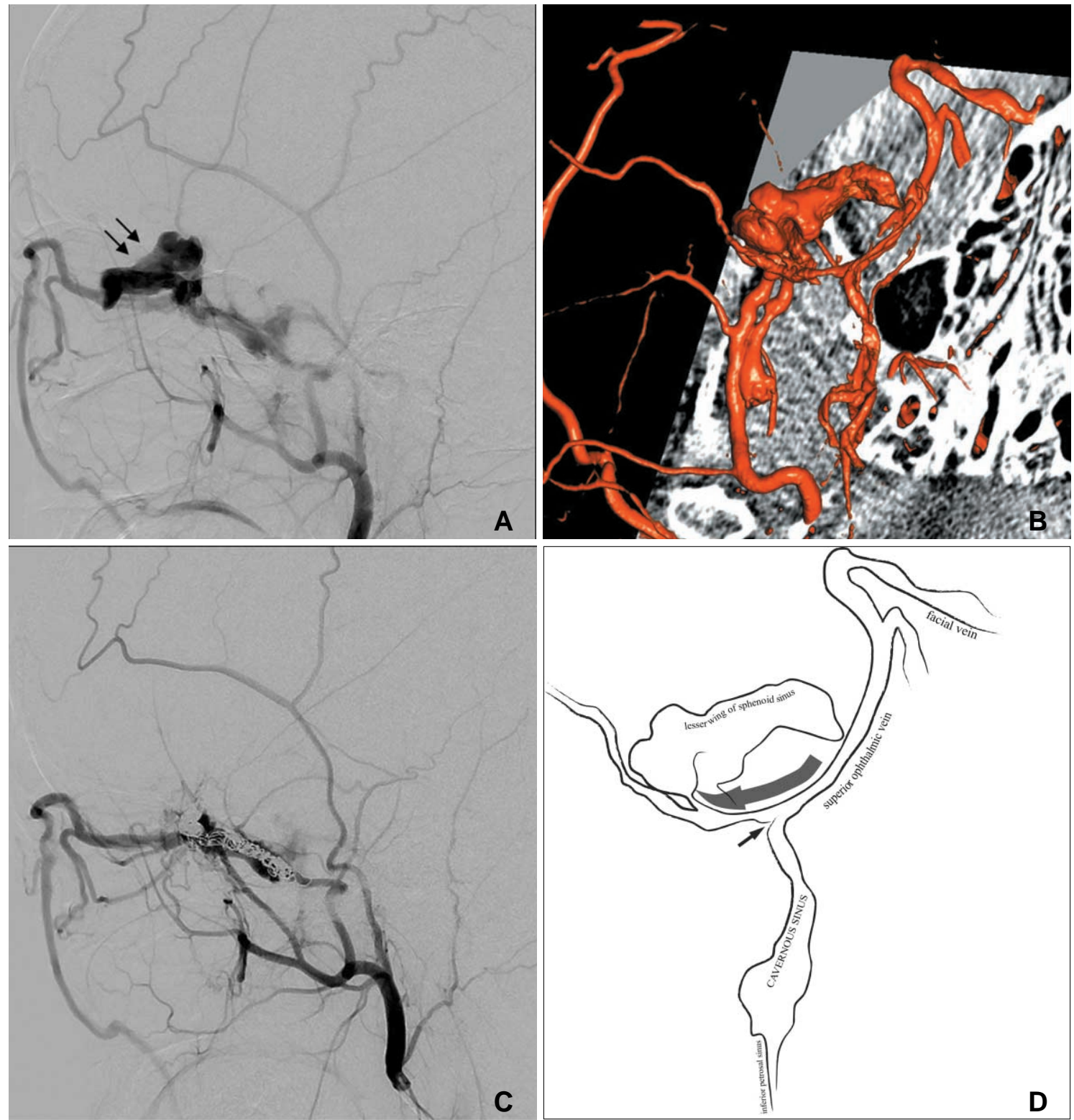

Fig. 2. Lateral view $(\mathbf{A})$ and three-dimensional reconstruction image $(\mathbf{B})$ of left ECA angiography showed DAVF with shunt flow into LWSS (arrows) connected with CS. The embolization for the lesion via arterial approach was performed using detachable and pushable fibered coils. (C) Follow-up angiography after 3 months showed recanalized shunt at the LWSS and venous drainage mainly into the SOV via the CS. The microcatheter was placed in the left IPS by microwire, and the left CS was selected. However, it could not be further advanced due to acute angle at the connection site (small arrow in figure $\mathbf{D}$ ) between the LWSS and CS.

Continued 

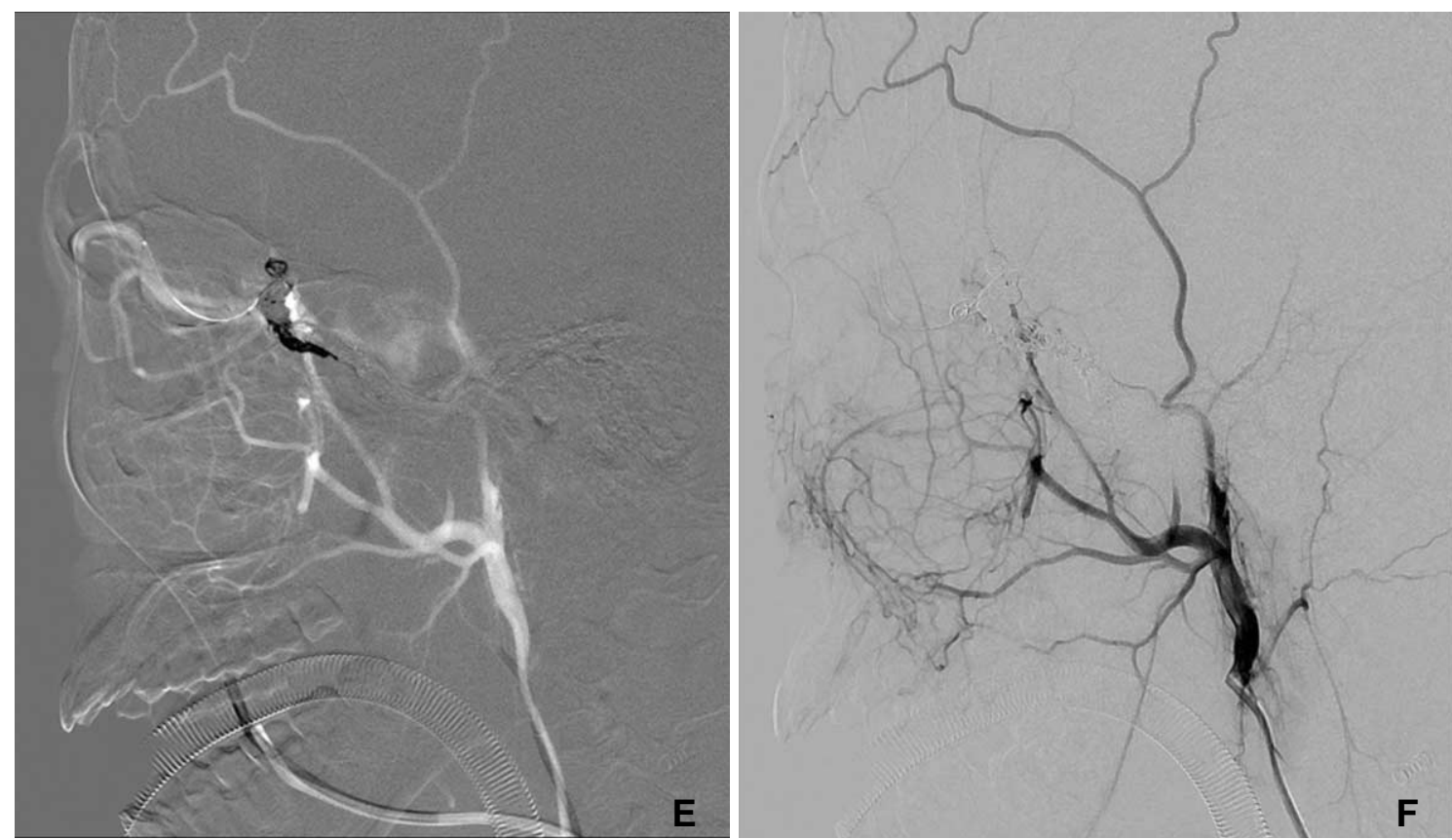

Fig. 2. (E) The microcatheter was placed in the left FV and SOV, and then it could be placed at the LWSS (through large arrow pathway in figure $\mathbf{D})$. (F) The remnant shunt was complete occluded by coils.

meningeal artery and performed embolization for the lesion via arterial approach using detachable and pushable fibered coils. The post-procedural angiography showed occlusion of internal maxillary artery but other fistula from ophthalmic artery was seen. The exophthalmos and chemosis improved in some degree after the procedure and the patient discharged, pledging to treat the remnant lesion again. After the initial treatment, follow-up angiography showed remained arterio-venous shunt at left LWSS, with arterial feeders including left middle meningeal artery and branch of inferolateral trunk from the left ICA, and venous drainage mainly into the SOV via left CS with small amount of venous drainage also into the left IPS. Under general anesthesia, initially a pre-shaped 45-degree microcatheter (Excelsior SL-10, Boston Scientific, Natick, MA, USA) was placed in the left middle meningeal artery. Liquid embolic agent (Onyx, Micro Therapeutics, Irvine, CA, USA) was prepared and injected through the microcatheter. The arteriovenous shunt from the left middle meningeal artery was completely occluded but the left LWSS region remained. We decided to approach for the fistula through venous route. A microcatheter was placed in the left IPS, and the left CS was selected. However, it could not be further advanced due to acute angle at the connection site between the left CS and LWSS. Other venous route was decided and we attempted to select FV. The microcatheter was placed in the left FV and
SOV, and then it was placed at the venous side near the arteriovenous shunt site at left LWSS. The remnant shunt was completely occluded by coils. The exophthalmos was resolved immediately after the procedure.

\section{DISCUSSION}

In this study, we reported favorable results and outcomes of trans-facial venous embolization for cavernous and paracavernous type DAVFs. The transvenous endovascular embolization is safe and effective option for DAVF requiring treatment, especially in DAVF with multiple feeders $[3,10,11]$. The transvenous route is usually through the IJV and the IPS up to the pathologic shunts of the CS [10]. Even though the IPS is partially or completely thrombosed, trans-IPS embolization of cavernous DAVF was often possible because of further developed materials and experience in the catheterization through the venous route [4]. Nevertheless, if the IPS route is not feasible or has failed in several efforts, an anterior transvenous approach to the CS through the SOV can be considerable and accessible as alternative route [12, 13]. In our cases, all except one case showed occluded IPS. However we challenged the other route and almost the procedures were successful. Therefore, even if the IPS as a shortest route to target lesion was inaccessible, the FV and SOV should be considerable route for the 
transvenous approach.

The anterior approach to the CS through the SOV can be performed through surgical exposure of SOV or the endovascular route (e.g., transfemoral transvenous) via the FV. The surgical approach for the SOV is usually performed after surgical exposure of the SOV or of the angular vein $[3,13,14]$. The SOV surgical route was described and reported by several authors $[3,15,16]$. In surgical approach for the FV or SOV routes, it require cut-down or puncture of the SOV and may remain cutaneous scars, which are not always cosmetically acceptable $[6,17]$. In addition, disadvantages and complications of the SOV exposure include bleeding from the SOV, difficulty in identifying the vein, injury of the supraorbital nerve and levator muscle, damage to the trochlea, infection, and granuloma $[6,8,17]$. Therefore, we supposed that transfemoral transvenous approach through the FV was more accessible and had more advantages than the surgical exposure of SOV for the treatment of cavernous DAVF in which trans-IPS approach is not accessible.

The attempted catheterization procedures are often very time-consuming. It would be beneficial to the patient as well as to the operator, especially in terms of radiation dose reduction, if the process of the transvenous catheterization procedure could be more easily performed. The efficacy of such procedures could very likely be improved if an appropriate venous route is identified and selected for catheterization at an early stage of the procedure [18]. Yu et al. [18] reported a venographic-based operational classification of cavernous type DAVF for early selection of the optimal venous route for embolization of the CS. They concluded that the knowledge of the status of compartmentalization of the CS as well as opacification of FV or IPS on angiogram were very important for the procedural success. In their study, anterior compartment of the CS was more accessible through the SOV, especially, with occluded IPS. Also in our study, all of cases showed opacification of FV and SOV on angiogram and all approaches through the FV and SOV were successful.

We experienced one case with DAVF in the region of the LWSS (Case 2). In general, asymptomatic DAVF of the LWSS probably do not require any correction [19]. However, Bithoh et al. [20] reported a case of DAVF of the LWSS in a patient with exophthalmos and chemosis due to venous hypertension in the SOV, which needed correction. Also in our case involving the LWSS, ophthalmic symptom presented as proptosis and chemosis. The LWSS is connected medially with the anterior and superior aspect of the CS [19]. The LWSS may be identified angiographically and distinguished from an ophthalmic vein, especially on lateral projections [19]. In the anteroposterior view, distinction between diploic vein of the LWSS and the SOV is made by identifying the characteristic inverted-S course of the SOV and its dorsal connection with the CS [19]. In these anatomical concepts of the LWSS and the CS, the LWSS locates on anterior compartment of the CS, far from the IPS. In addition, the bridge between the LWSS and the CS may have unusual angle if the approach is performed through the IPS. As expected, our case involving the LWSS was not accessible through the IPS due to bizarre course from the CS to the LWSS. Finally, we challenged the approach to the LWSS through the FV and SOV and the attempt was successful.

In conclusion, in the endovascular treatment of cavernous or paracavernous DAVF in which trans-IPS approach is not feasible, the FV provides safe and effective treatment route for transvenous embolization. Especially, in anterior or superior compartmental lesion, the transfacial-ophthalmic venous approach may be feasible and effective modality. In addition, the compartmental venous anatomy should be considered and identified for proper targeting the lesions.

\section{Acknowledgments}

The author thanks Joo-yong Ahn for technical assistance of angiographic machinery during our procedures in Seoul St. Mary's hospital.

\section{References}

1. Agid R, Willinsky RA, Haw C, Souza MP, Vanek IJ, terBrugge KG. Targeted compartmental embolization of cavernous sinus dural arteriovenous fistulae using transfemoral medial and lateral facial vein approaches. Neuroradiology 2004;46:156-160

2. Kim DJ, Kim DI, Suh SH, Kim J, Lee SK, Kim EY, et al. Results of transvenous embolization of cavernous dural arteriovenous fistula: a single-center experience with emphasis on complications and management. AJNR Am J Neuroradiol 2006;27:2078-2082

3. Biondi A, Milea D, Cognard C, Ricciardi GK, Bonneville F, van Effenterre R. Cavernous sinus dural fistulae treated by transvenous approach through the facial vein: report of seven cases and review of the literature. AJNR Am J Neuroradiol 2003;24:12401246

4. Benndorf G, Bender A, Lehmann R, Lanksch W. Transvenous occlusion of dural cavernous sinus fistulas through the thrombosed inferior petrosal sinus: report of four cases and review of the literature. Surg Neurol 2000;54:42-54

5. Elhammady MS, Peterson EC, Aziz-Sultan MA. Onyx embolization of a carotid cavernous fistula via direct transorbital puncture. J Neurosurg 2011;114:129-132

6. Quiñones D, Duckwiler G, Gobin PY, Goldberg RA, Viñuela F. 


\section{Myeong Jin Kim, et al.}

Embolization of dural cavernous fistulas via superior ophthalmic vein approach. AJNR Am J Neuroradiol 1997;18:921-928

7. Venturi C, Bracco S, Cerase A, Gennari P, Lorè F, Polito E, et al. Endovascular treatment of a cavernous sinus dural arteriovenous fistula by transvenous embolisation through the superior ophthalmic vein via cannulation of a frontal vein. Neuroradiology 2003;45:574-578

8. Oishi H, Arai H, Sato K, Iizuka Y. Complications associated with transvenous embolisation of cavernous dural arteriovenous fistula. Acta Neurochir (Wien) 1999;141:1265-1271

9. Ha JG, Jeong HW, In HS, Choi SJ. Transvenous embolization of cavernous sinus dural arteriovenous fistula using the direct superior ophthalmic vein approach: a case report. Neurointervention 2011;6:100-103

10. Liu HM, Wang YH, Chen YF, Cheng JS, Yip PK, Tu YK. Longterm clinical outcome of spontaneous carotid cavernous sinus fistulae supplied by dural branches of the internal carotid artery. Neuroradiology 2001;43:1007-1014

11. Meyers PM, Halbach VV, Dowd CF, Lempert TE, Malek AM, Phatouros CC, et al. Dural carotid cavernous fistula: definitive endovascular management and long-term follow-up. Am J Ophthalmol 2002;134:85-92

12. Benndorf G, Bender A, Campi A, Menneking H, Lanksch WR. Treatment of a cavernous sinus dural arteriovenous fistula by deep orbital puncture of the superior ophthalmic vein. Neuroradiology 2001;43:499-502

13. Berlis A, Klisch J, Spetzger U, Faist M, Schumacher M. Carotid cavernous fistula: embolization via a bilateral superior ophthalmic vein approach. AJNR Am J Neuroradiol 2002;23:1736-1738

14. Derang J, Ying H, Long Y, Reifa S, Qiming W, Yimu F, et al. Treatment of carotid-cavernous sinus fistulas retrograde via the superior ophthalmic vein (SOV). Surg Neurol 1999;52:286-292

15. Courtheoux P, Huet H, Tournade A, Theron J. Dural fistula of the cavernous sinus. Treatment through an intravenous approach apropos of 4 cases. J Neuroradiol 1995;22:301-308

16. Dashti SR, Fiorella D, Spetzler RF, Albuquerque FC, McDougall CG. Transorbital endovascular embolization of dural carotidcavernous fistula: access to cavernous sinus through direct puncture: case examples and technical report. Neurosurgery 2011;68:75-83

17. Goldberg RA, Goldey SH, Duckwiler G, Vinuela F. Management of cavernous sinus-dural fistulas. Indications and techniques for primary embolization via the superior ophthalmic vein. Arch Ophthalmol 1996;114:707-714

18. Yu SC, Cheng KM, Tam PH, Wong GK, Chan CM, Cheung YL, et al. A venographic operational classification for transvenous embolization of dural carotid-cavernous fistula. Neuroradiology 2011;53:993-999

19. San Millan Ruiz D, Fasel JH, Rufenacht DA, Gailloud P. The sphenoparietal sinus of breschet: does it exist? An anatomic study. AJNR Am J Neuroradiol 2004;25:112-120

20. Bitoh S, Arita N, Fujiwara M, Ozaki K, Nakao Y. Dural arteriovenous malformation near the left sphenoparietal sinus. Surg Neurol 1980;13:345-349 